\title{
Field optimisation of MosquiTRAP sampling for monitoring Aedes aegypti Linnaeus (Diptera: Culicidae)
}

\author{
Marcelo Carvalho de Resende ${ }^{2} /{ }^{+}$, Tatiana Mingote Ferreira de Ázara ${ }^{1}$, Ione Oliveira Costa ${ }^{3}$, \\ Laila Costa Heringer ${ }^{1}$, Mateus Ramos de Andrade', José Luiz Acebal ${ }^{4}$, Álvaro Eduardo Eiras ${ }^{1}$
}

\author{
${ }^{1}$ Laboratório de Ecologia Química de Insetos Vetores, Departamento de Parasitologia, Universidade Federal de Minas Gerais, \\ Av. Antônio Carlos 6627, 31.270-901 Belo Horizonte, MG, Brasil ${ }^{2}$ Serviço de Saúde Ambiental, Fundação Nacional da Saúde, \\ Ministério da Saúde, Belo Horizonte, MG, Brasil ${ }^{3}$ Centro de Controle de Zoonoses Regional Oeste, \\ Prefeitura de Belo Horizonte, Belo Horizonte, MG, Brasil ${ }^{4}$ Departamento de Física e Matemática, \\ Centro Federal de Educação Tecnológica de Minas Gerais, Belo Horizonte, MG, Brasil
}

A sticky trap designed to capture gravid Aedes (Stegomyia) aegypti mosquitoes, MosquiTRAP, has been evaluated for monitoring this species in Brazil. However, the effects of trap densities on the capture rate of Ae. aegypti females and the sensitivity of vector detection are still unknown. After a preliminary study has identified areas of high and low female mosquito abundance, a set of experiments was conducted in four neighbourhoods of Belo Horizonte (state of Minas Gerais, Brazil) using densities of 1, 2, 4, 8, 16, 32 and 64 traps per block. Trap sensitivity (positive MosquiTRAP index) increased significantly when 1-8 MosquiTRAPs were installed per block in both high and low abundance areas. A strong fit was obtained for the total number of mosquitoes captured with increasing trap densities through a non-linear function (Box-Lucas) $\left(r^{2}=0,994\right)$, which likely exhibits saturation towards an equilibrium level. The capacity of the Mean Female Aedes Index to distinguish between areas of high and low Ae. aegypti abundance was also investigated; the achieved differentiation was shown to be dependent on the MosquiTRAP density.

Key words: Aedes aegypti - sticky trap - trap density - Box-Lucas function

Dengue fever has become an increasingly significant health threat in Brazil as well as worldwide. Vector control, which is available in many forms, remains a key strategy for dengue fever prevention. Among the instruments available to assess vector control interventions, the traditional Stegomyia indices proposed by Connor and Monroe (1923) and Breteau (1954) have operational value and are a common type of measurement used for directing vector control efforts. Nevertheless, there are shortcomings associated with these methods because they are based on surveying immature stages (larvae and/or pupae) of Aedes aegypti mosquitoes (Diptera: Culicidae), which makes them inadequate for determining transmission risk. Adult mosquito abundance is considered to be the most appropriate stage at which to assess transmission risk (Focks 2003) and the development of new entomological indicators that reflect the potential for the transmission of the dengue virus would be beneficial for monitoring programs and vector control interventions (Focks \& Chadee 1997, Focks 2003, Teixeira et al. 2005).

In Brazil, surveillance of Ae aegypti is usually performed by sampling larvae and pupae in dwellings and buildings (MS 2002, 2009). However, this method has a low sensitivity and is laborious because it requires search-

Financial support: $\mathrm{CNPc}$

+ Corresponding author: marcelo.resende@funasa.gov.br Received 17 December 2010

Accepted 15 February 2012 ing for breeding sites in urban areas and then identifying the field-collected mosquitoes in the laboratory (Braga et al. 2000, Braga \& Valle 2008, Eiras \& Resende 2009).

As a result of these limitations, a sticky trap known as MosquiTRAP ${ }^{\mathrm{TM}}$ was developed to facilitate the capture of Ae. aegypti adult mosquitoes, mainly gravid females, using a synthetic oviposition attractant (AtrAedes ${ }^{\mathrm{TM}}$ ) (Fávaro et al. 2006, Maciel-de-Freitas et al. 2006, Eiras \& Resende 2009). The trap allows immediate identification of captured mosquitoes at the time of inspection; thus, MosquiTRAP $^{\mathrm{TM}}$ avoids the time and labour required for laboratory identification of mosquitoes (Fávaro et al. 2006, Gama et al. 2007, Eiras \& Resende 2009). It is also an effective device for trapping Ae. aegypti when larval surveys fail to detect the presence of this species (Gama et al. 2007). Recently, this sticky trap has been used in a variety of investigations, including estimates of the size of the Ae. aegypti female population (Maciel-de-Freitas et al. 2008) or the temporal distribution of Ae. aegypti populations (Honório et al. 2009a), spatial evaluations and modelling of dengue vector density (Honório et al. 2009b), comparative studies between MosquitoTrap ${ }^{\mathrm{TM}}$ and other sampling methods, including larval and oviposition surveillance (Gama et al. 2007, Lorenço-deOliveira et al. 2008), Nasci (Fávaro et al. 2008) and back-pack aspirators (Maciel-de-Freitas et al. 2006), and monitoring of Ae. aegypti females (Ordónez-Gonzalez et al. 2001, Russell \& Ritchie 2004, Fávaro et al. 2006, Facchinelli et al. 2007, Eiras \& Resende 2009).

The entomological indices provided by MosquiTRAP $^{\mathrm{TM}}$, the Mean Female Aedes Index (MFAI) and the Positivity of MosquiTRAP ${ }^{\mathrm{TM}}$ Index (PMI), are 
currently being used as potential entomological indices for monitoring adult Ae. aegypti populations in Brazil (WHO 2006) using a density of one sticky trap/200 $\mathrm{m}^{2}$ (Eiras \& Resende 2009). However, the effectiveness of this trap density and the sensitivity of the MosquiTRAP ${ }^{\mathrm{TM}}$ indices at different trap densities have not yet been reported.

Therefore, this study was designed to answer four research questions: (i) What is the effect of using different trap densities per block and per house on the capture rate of Ae. aegypti females? (ii) How do the entomological indices provided by MosquiTRAP ${ }^{\mathrm{TM}}$ behave when different trap densities are used? (iii) What is the sensitivity of MosquiTRAP ${ }^{\mathrm{TM}}$ in detecting the vector in the area of study? (iv) Do trap captures reach saturation when increased trap densities are used?

\section{MATERIALS AND METHODS}

Experimental area - The study was carried out in the West Sanitary District (WSD) of the city of Belo Horizonte, in the state of Minas Gerais (MG), Brazil, as defined by the regional administration. This district has a population of 268,124 according to data provided by the Brazilian Institute of Geography and Statistics (IBGE 2000), an area of $31.3 \mathrm{Km}^{2}$ and a population density of 8,573 persons $/ \mathrm{Km}^{2}$. Based on information regarding the presence of Ae. aegypti provided by the Centre of Zoonosis Control (CZC) of WSD (PBH 2009), four WSD neighbourhoods were selected for the present study. The four neighbourhoods (Noraldino, São Jorge A, São Jorge $\mathrm{B}$ and Ventosa) are characterised by the presence of brick houses, paved streets, weekly garbage collection and a regular water supply system.

MosquiTRAP ${ }^{T M}$ - The investigated sticky trap, MosquiTRAP $^{\mathrm{TM}}$ (v. 3.0, Ecovec SA, Belo Horizonte, MG, Brazil), as described by Eiras and Resende (2009), consists of a matte black polyethylene container $(33 \mathrm{~cm}$ high and $15 \mathrm{~cm}$ in diameter) divided into two parts: a lower base filled with approximately $300 \mathrm{~mL}$ of water and an upper part with a funnel-shaped opening that facilitates the mosquitoes' entry and hinders their exit. A sticky card is attached at the water line from the lower base to the upper entrance of the trap. A synthetic oviposition attractant (AtrAedes) is attached to the sticky card to attract and capture ovipositing adult female mosquitoes. Sticky traps were installed at visible sites outdoors (Fávaro et al. 2006) in either the front or back yards of houses at a maximum height of $1.5 \mathrm{~m}$, protected from the sun and rain and out of reach of children and domestic animals. Ten health agents worked in pairs and were provided training regarding MosquiTRAP ${ }^{\mathrm{TM}}$ assembly and installation procedures, identification of trapped mosquitoes and inspection of the traps.

Methods - The study was divided into two stages: (i) a preliminary analysis to categorise the two experimental groups, i.e., areas of high Ae. aegypti mosquito abundance and areas of low Ae. aegypti mosquito abundance, and (ii) an evaluation of the use of different trap densities for capturing Ae. aegypti females.

The preliminary analysis was conducted for three days and consisted of placing a total of 120 traps in the four study neighbourhoods (30 blocks/neighbourhood), for a density of one trap per block. The number of $A e$. aegypti females caught in each sticky trap was recorded after three days. Based on this preliminary survey, two groups, each consisting of seven blocks, were delineated for each of the four neighbourhoods: (i) high abundancearea (i.e., at least one Ae. aegypti female was captured during the preliminary survey) and (ii) a low abundance area (i.e., no evidence of Ae. aegypti detected during the preliminary survey).

The evaluation of trap density was performed over four weeks and included 14 blocks per neighbourhood (as described above), for a total of 56 blocks among the four neighbourhoods. Trap inspections were conducted weekly and the 56 blocks were randomly assigned to receive $1,2,4,8,16,32$ or 64 sticky traps per block. In the blocks receiving one-16 sticky traps, each house was provided with only one sticky trap, whereas blocks with 32 or 64 traps had two and four traps in each house, respectively. Thus, a total of 126 houses (63 for

TABLE

Distribution of MosquiTRAP per block and dwelling in either low or high abundance experimental areas of each neighbourhood of Belo Horizonte, state of Minas Gerais, Brazil

\begin{tabular}{ccccc}
\hline \multirow{2}{*}{$\begin{array}{c}\text { Traps per block } \\
\text { (n) }\end{array}$} & Houses with trap & Traps per house & & \multicolumn{2}{c}{ Mean number of houses per block in each density $(\mathrm{x} \pm \mathrm{SE})$} \\
\cline { 4 - 5 }$(\mathrm{n})$ & 1 & 1 & High abundance & Low abundance \\
\hline 1 & 1 & 1 & $24 \pm 15.0$ & $21 \pm 11.3$ \\
2 & 2 & 1 & $29 \pm 8.5$ & $40 \pm 7.9$ \\
4 & 4 & $\mathbf{1}$ & $33 \pm 19.1$ & $43 \pm 9.1$ \\
8 & 8 & $\mathbf{2}$ & $33 \pm 10.0$ & $32 \pm 7.9$ \\
$\mathbf{1 6}$ & $\mathbf{1 6}$ & $\mathbf{3 4}$ & $\mathbf{5 0} \pm \mathbf{1 9 . 7}$ & $\mathbf{3 4} \pm \mathbf{1 6 . 0}$ \\
$\mathbf{3 2}$ & $\mathbf{1 6}$ & $\mathbf{4}$ & $\mathbf{4 9} \pm \mathbf{2 9 . 4}$ & $\mathbf{3 7} \pm \mathbf{1 2 . 0}$ \\
$\mathbf{6 4}$ & $\mathbf{1 6}$ & &
\end{tabular}

area in bold represents the number MosquiTRAP (i.e. 1,2 and 4) placed per house that received separate analysis; SE: standard error. 
high abundance areas and 63 for low abundance areas) were sampled per neighbourhood (Table). For the blocks that received more than one sticky trap, we distributed an equal number of sticky traps along the four sides of each block. The distance between blocks in each neighbourhood typically varied from 100-300 m and the distance between the neighbourhoods was at least $500 \mathrm{~m}$. Although the distribution of houses was similar in the experimental areas, the mean number of homes varied from 22-44 houses per block (Table).

The sticky traps were inspected weekly by pairs of health agents (field workers) provided by the CZC-WHD of Belo Horizonte, as well as by trainees of the Laboratory of Chemical Ecology of Insect Vectors in the Department of Parasitology of the Federal University of Minas Gerais. During the weekly surveys, the adult Ae. aegypti mosquitoes caught in the sticky traps were identified in the field under a 20X magnifying glass and data were recorded on worksheets.

The entomological surveillance parameters, whose averages were evaluated over a four-week time basis, were as follows.

Total number of Ae. aegypti females captured based on trap density - The total number of Ae. aegypti females captured in each block was calculated to evaluate whether the trap captures were affected by the density of sticky traps placed in each block and/or house.

$P M I$ - The capacity of the traps to detect the presence of Ae. aegypti in each block was assessed (i.e., the sticky trap was considered positive for the vector when at least one specimen of Ae. aegypti was captured per block). Trap positivity was calculated using the following formula: PMI = total number of positive blocks for Ae. aegypti/total number of blocks monitored with a sticky trap x 100 (Eiras \& Resende 2009).

Effect of MosquiTRAPTM density per house - This index was used to evaluate the number of Ae. aegypti females caught per inspected house within blocks that had trap densities of 16, 32 and 64 MosquiTRAPs $^{\mathrm{TM}}$ per block, which was equivalent to one, two and four sticky traps per house, respectively.

$M F A I$ - This index consisted of the number of $A e$. aegypti females captured divided by the total number of sticky traps inspected within the block and divided by the number of weeks monitored (Eiras \& Resende 2009).

Mean Female Aedes per House Index (MFAHI) This index was calculated as the number of Ae. aegypti females caught divided by the number of weeks monitored divided by the number of sticky traps and by the mean number of houses within each density assessed.

Statistical analysis - The number of females captured per trap in low and high abundance areas and both types of areas together (overall) was subjected to the D'Agostino-Pearson normality test, followed by ANOVA and then Tukey's test (Sokal \& Rolf 1995) to assess the MFAHI (GraphPad Prism v. 5.01) and the effect of the MosquiTRAP ${ }^{\mathrm{TM}}$ density per house. The MFAI entomological index was subjected to Kruskal-Wallis and/ or Mann-Whitney tests. The chi-squared test was used to determine whether there were significant differences between neighbourhoods and high and low-mosquito abundance areas. The Box-Lucas function (Box \& Lucas 1959) was used to fit the total number of Ae. aegypti females caught based on trap density within the low and high abundance areas and the MFAHI (OriginLab 6.0, Northampton, MA, USA). To assess the PMI, data were subjected to a Kruskal-Wallis test followed by Dunn's multiple comparison test. The statistical frequency distribution for the number of MosquiTRAP ${ }^{\mathrm{TM}}$ captures was fitted with a Poisson distribution (Matlab R2007b, Natick, MA, USA). The total, as well as the mean number of Ae. aegypti females captured per house and per block for each trap density studied, were analysed through regression analysis.

\section{RESULTS}

Preliminary study to categorise high and low Ae. aegypti mosquito abundance areas - A total of 1,914 Ae. aegypti females were captured in the four study neighbourhoods during the preliminary analysis conducted to categorise areas of high and low abundance. Although the Noraldino neighbourhood showed a higher number of captures compared with São Jorge A, São Jorge B and Ventosa $(612,451,423$ and 428, respectively), no significant difference was observed between neighbourhoods $\left(\chi^{2}=1.200 ; p=0.2733\right)$. However, there was a significant difference between areas within neighbourhoods delineated with high and low mosquito abundances $\left(\chi^{2}\right.$ $=15.221 ; \mathrm{p}=0.0016$ ).

Total number of Ae. aegypti females captured based on trap density - The total number of Ae. aegypti females captured per block ranged from 21, when one MosquiTRAPTM was used per block to a maximum of 871 females, when 64 MosquiTRAPs ${ }^{\mathrm{TM}}$ were installed per block. When the density was increased from foureight MosquiTRAP ${ }^{\mathrm{Ts} \mathrm{M}}$ per block, a $27.7 \%$ increase in the capture of Ae. aegypti females was observed. When the density was raised from eight-16 MosquiTRAPs ${ }^{\mathrm{TM}}$ per block and from 16-32 traps per block, the capture rates increased by $133 \%$ and $121.5 \%$, respectively.

During the four-week study, the results showed that the total number of Ae. aegypti females captured increased with increasing trap densities in both high and low abundance areas. A linear relationship with a good fit $\left(\mathrm{r}^{2}=0.985\right)$ was observed between the trap density and the total number of Ae. aegypti females captured in both high and low abundance areas.

A better-fitting model $\left(\mathrm{r}^{2}=0.9939\right)$ (Fig. 1A) was obtained through the Box-Lucas function, $\mathrm{N}(\mathrm{D})=\mathrm{a}[1-$ $\left.\mathrm{e}^{-\mathrm{bD}}\right],(\mathrm{a}>0, \mathrm{~b}>0)$, for mosquito captures according to trap density in high, low and overall (i.e., high and low combined) abundance areas. In this model, parameter a represents an equilibrium level at which the number of captures $(\mathrm{N})$ becomes saturated with increasing density (D) and parameter $\mathrm{b}$ provides an estimate of the rate at which this will occur. The greater the value of $b$, the more rapidly saturation is obtained with increasing trap densities. The Box-Lucas saturation function also fit the data for high $\left(r^{2}=0.9903\right)$ and low $\left(r^{2}=0.9917\right)$ abundance areas (Fig. 1B, C). In low abundance areas, the number 
of captures reached values close to the equilibrium level of the Box-Lucas function, which contrasted with what was observed for high abundance areas over the studied range of sticky trap densities.

$P M I$ - As expected, the number of mosquito captures per block increased with increasing trap density. The positivity of sticky traps in both high and low abundance areas also increased significantly as higher densities of MosquiTRAPs ${ }^{\mathrm{TM}}$ were installed per block (Kruskal-Wallis, $\mathrm{H}=66.6, \mathrm{p}<0.01$ ). This correlation was found in areas preliminarily classified as both high abundance $(\mathrm{H}=18.5, \mathrm{p}<0.01)$ and low abundance areas $(H=22.0, p<0.01)$ (Fig. 2A, B).

In blocks where only one MosquiTRAP ${ }^{\mathrm{TM}}$ was installed, the value of PMI was $31.3 \%$ in of low abundance areas and $50 \%$ in high abundance areas. The maximum rate of detection (100\%) of Ae. aegypti females in the four neighbourhoods was observed for the overall area when 16, 32 and 64 sticky traps were installed per block (Fig. 2A, B). The mean PMI observed for densities of one and two sticky traps per block in high (Fig. 2A) and low abundance (Fig. 2B) areas was significantly lower than that observed for densities of eight or more traps per block.

Effect of MosquiTRAP ${ }^{T M}$ density per house - The mean number of Ae. aegypti females caught per house varied significantly (ANOVA; $\mathrm{F}_{247}=30.7 ; \mathrm{p}<0.01$ ) when one, two or four sticky traps were installed per house, corresponding to densities of 16, 32 and 64 traps per block, respectively (Fig. 3, Table). The mean number of Ae. aegypti females caught increased with an increasing number of sticky traps per house. However, the relative increase in captures from one-two sticky traps per house was $122.1 \%$ greater than that from two-four sticky traps per house, which was $59.1 \%$.

MFAI - For areas of combined high and low Ae. aegypti abundance (i.e., overall), higher MFAI and variance values were observed at densities of one, two and four sticky traps per block, while lower values of the MFAI and variance were observed at trap densities ranging from eight- 64 traps per block. This index varied from 0.41-0.66 for densities of one-four traps per block, respectively. There was no significant difference detected in the average number of mosquitoes captured per block based on trap density (Fig. 4A) (KruskalWallis, $\mathrm{H}=6.378 ; \mathrm{p}=0.3829$ ).

When low and high abundance areas were analysed separately, the MFAI ranged from 0.94-0.39 for high abundance areas, when from one-eight traps were installed per block (Fig. 4B); whereas for low abundance areas (Fig. 4C) the MFAI ranged from 0.66-0.28 when from two-64 traps per block were installed. There was no significant the difference detected in the MFAI (Fig. $4 \mathrm{~B}, \mathrm{C})$ for the sticky trap density variation per block between low $(\mathrm{H}=8.195 ; \mathrm{p}=0.2241)$ and high abundance areas $(H=5.128 ; p=0.5275)$. Nevertheless, when high and low abundance areas were compared, regardless of the trap density per block, the MFAI was significantly different (Mann-Whitney test, $\mathrm{U}=5234 ; \mathrm{p}=0.0315$ ). Upon analysing the MFAI behaviour in regard to the
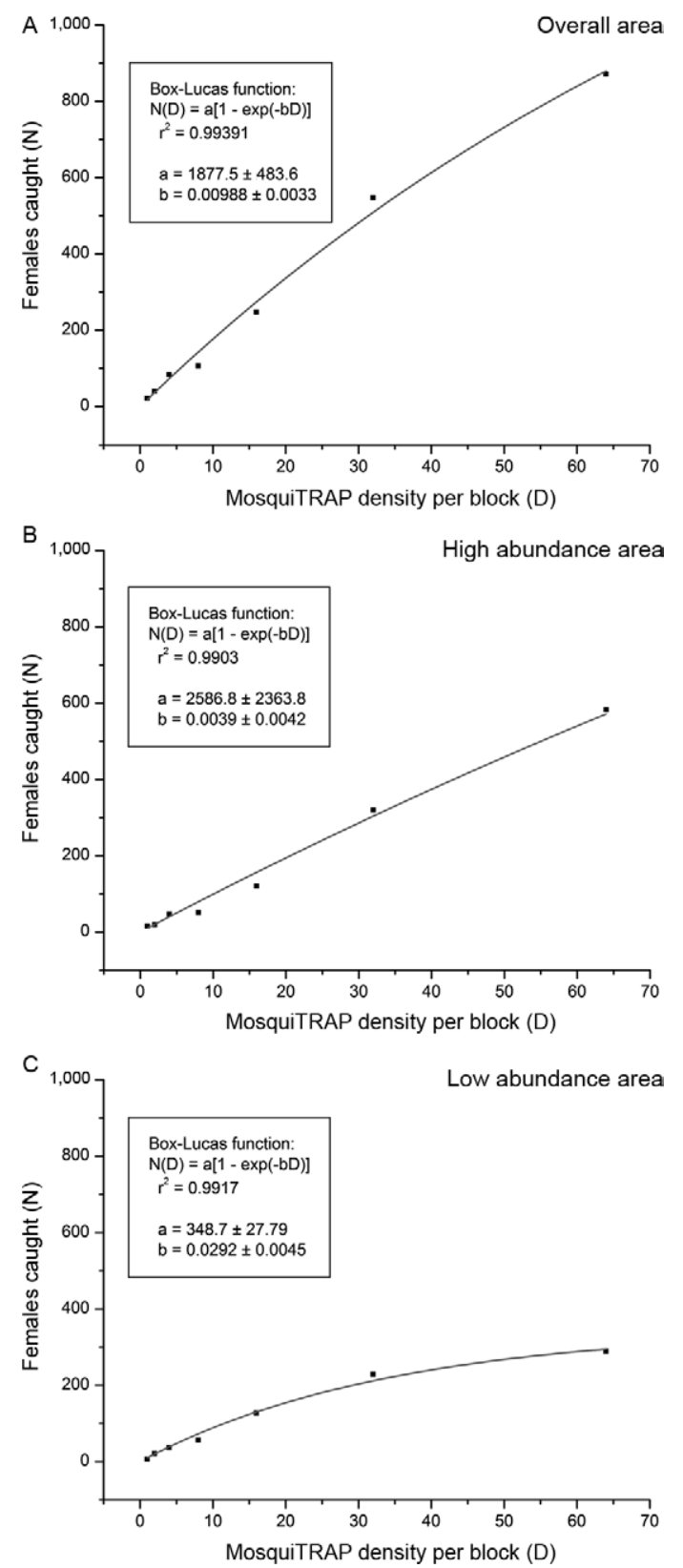

Fig. 1: total number of Aedes aegypti female captured by using different densities of MosquiTRAP in (A) overall abundance, (B) high abundance and (C) low abundance areas and their non-linear fitness set by Box-Lucas function, Belo Horizonte, Minas Gerais, Brazil.

possibility of differentiating high abundance areas from low abundance areas for each trap density studied, the p-values obtained were $0.0006,0.0519$ and 0.1587 for 64 , 32 and one traps per block, respectively. For the remaining densities, $\mathrm{p} \geq 0.5275$.

To investigate the higher variance as well the higher number of zero captures obtained, the Poisson distribution was used to represent the MosquiTRAP ${ }^{\mathrm{TM}}$ capture frequency. For one trap per block, in both high and low abundance areas, the fit of the Poisson distribution to the 

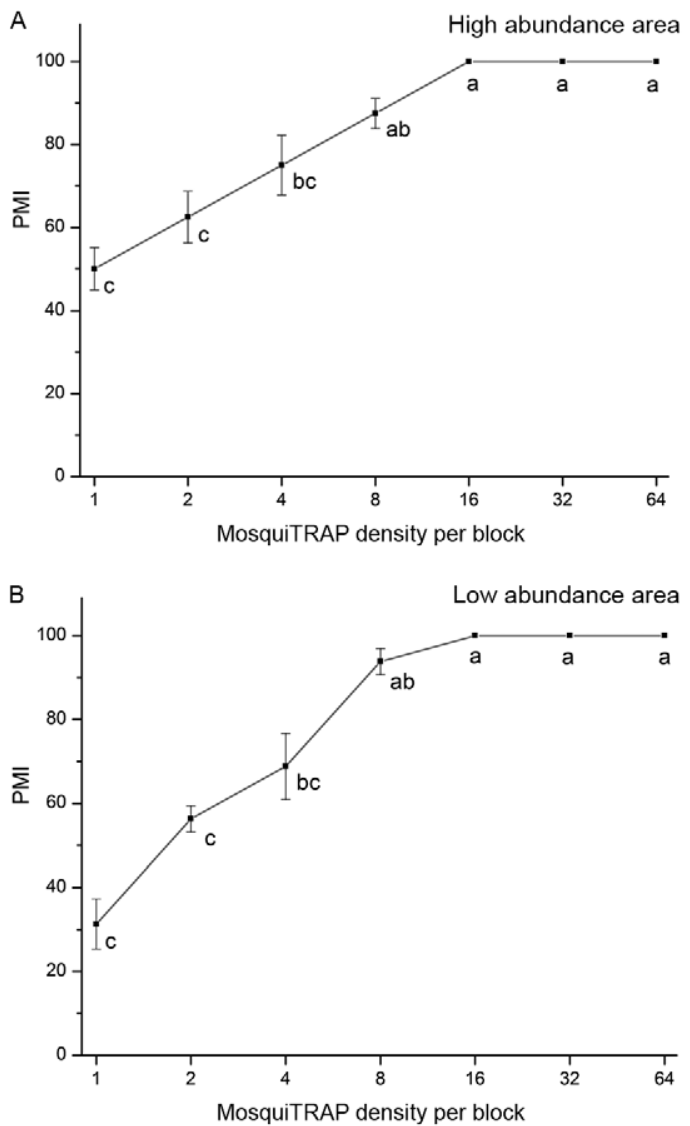

Fig. 2: positivity of MosquiTRAP Index (PMI) at different trap densities in Belo Horizonte, Minas Gerais, Brazil. The different letters indicate significant difference (Tukey $\mathrm{p}<0.05$ ).

frequency of the number of captures was good $\left(\mathrm{r}^{2}=0.999\right.$ and $\mathrm{r}^{2}=0.765$, respectively). For two traps per block, the Poisson distribution also fit the frequency of the number of captures for both low $\left(r^{2}=0.926\right)$ and high $\left(r^{2}=0.915\right)$ abundance areas.

MFAHI - In general, the MFAHI exhibited the same pattern based on the total number of Ae. aegypti females captured using different MosquiTRAP ${ }^{\mathrm{TM}}$ densities (Fig. $5 \mathrm{~A})$. The Box-Lucas function described above again showed a strong goodness of fit $\left(\mathrm{r}^{2}=0.995\right)$, with the mean number of Ae. aegypti captures increasing as the trap density increased. A significant difference in the MFAHI (Fig. 5A) based on sticky trap density was observed (ANOVA: $\mathrm{F}_{[6,217]}=65.84 ; \mathrm{p}<0.0001$ ) for the overall area. Smaller variance values were observed for trap densities of 16, 32 and 64 compared to trap densities of one to eight. For the high and low abundance areas, there was significant variation between the MFAHI (Fig. 5B, C) and trap density (ANOVA: $\mathrm{F}_{[6.217]}=38.23 ; \mathrm{p}<0.0001$ and ANOVA: $\mathrm{F}_{[6,217]}=53.69 ; \mathrm{p}<0.0001$, respectively).

\section{DISCUSSION}

Although MosquiTRAPs ${ }^{\mathrm{TM}}$ have been used for weekly monitoring of the dengue vector, some studies (Fávaro et al. 2006, Maciel-Freitas et al. 2006, Gama et al. 2007,

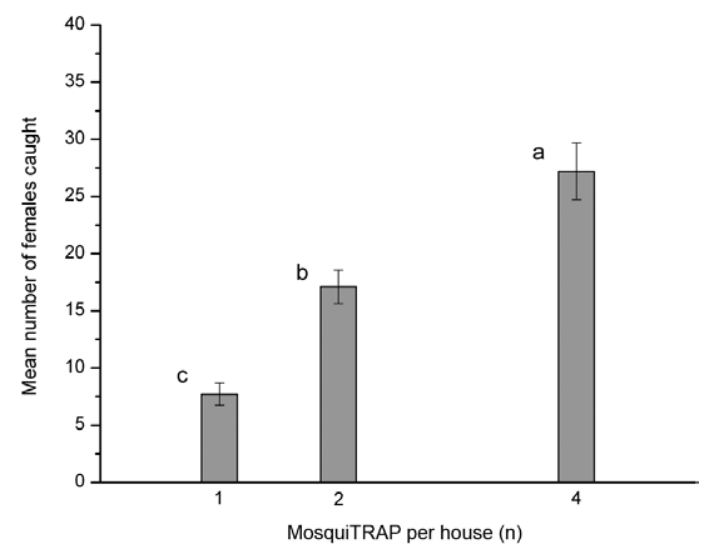

Fig. 3: effect of MosquiTRAP density per house in Belo Horizonte, Minas Gerais, Brazil. The different letters indicate significant difference (Tukey $\mathrm{p}<0.05$ ).

Eiras \& Resende 2009) suggest that there is no evidence that weekly trap inspection monitoring is ideal. The weekly basis of the present survey was chosen due to operational and logistic factors related to the availability of field workers (health agents). For example, one field worker can assess larger areas and a higher number of traps by maintaining weekly maintaining weekly visits. It can be expected that shorter periods of surveillance might result in a decrease in the recorded trap sensitivity, whereas longer periods of surveillance might contribute to the detection of increases in variance.

Several studies focused on determining optimal trap densities have been reported for agricultural pests, such as fruit flies (Iga 1982) and moths (Faccioli et al. 1993), but for Ae. Aegypti the optimal trap densities when using MosquiTRAP ${ }^{\mathrm{TM}}$ in urban areas remain unknown.

It is well known that populations of Ae. aegypti are not distributed homogeneously in the field (Lagrotta et al. 2008). Instead, they are probably dispersed in clusters (Eiras \& Resende 2009, Honório et al. 2009a) at different distances (Reiter et al. 1995, Honório et al. 2003). In the present investigation, significant differences were detected between the subsets of high and low Ae. aegypti female abundances within neighbourhoods, despite using a preliminary surveillance period of only three days in the field, which is a relatively short period of time.

Although the linear regression showed an extremely good fit $\left(\mathrm{r}^{2}=0.985\right)$ for the total number of $A e$. aegypti females caught overall (i.e., combined high and low abundance areas), we also investigated an alternative non-linear function (the Box-Lucas function), which showed an even higher correlation ranging from $r^{2}=0.9903-r^{2}=0.9939$, depending on the level of mosquito abundance investigated. Parameter a of the Box-Lucas function corresponds to the equilibrium level of the total number of mosquitoes potentially available for capture as the density of sticky traps becomes sufficiently high. The number of Ae. aegypti females caught in the low abundance areas (28 blocks total) reached values close to the Box-Lucas function equilibrium level $(\mathrm{a}=348.7$ \pm 27.79 ), which contrasts what was observed for the high 

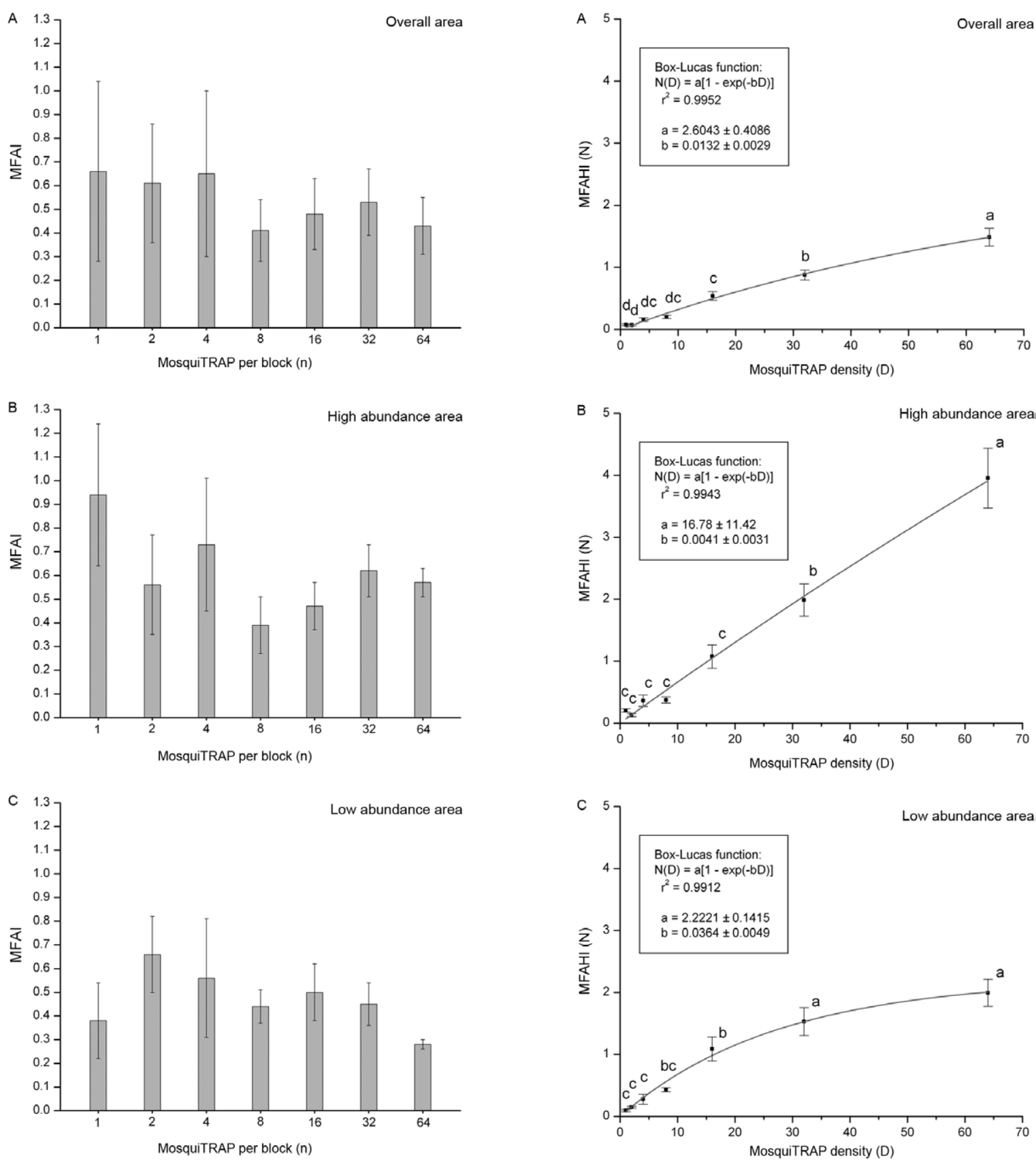

Fig. 4: mean Female of Aedes Index (MFAI) at different MosquiTRAP densities per block in (A) overall abundance (B) high abundance and (C) low abundance areas in Belo Horizonte, Minas Gerais, Brazil. The different letters indicate significant difference (Tukey $\mathrm{p}<0.05$ ).

abundance areas ( 28 blocks total) $(\mathrm{a}=2,586.8 \pm 2,363.8)$ and the total number in the overall areas $(\mathrm{a}=1,877.5$ \pm 483.6 ), which clearly did not reach values close to the equilibrium level based on the available dataset. The graphing trends in those cases showed similar patterns. Parameter $b$, which is equally as important as the equilibrium level (a) provides a measure of how rapidly the number of captures approaches the equilibrium level, a,

as the density of sticky traps increases. The higher the value of $b$ is, the more rapidly the function approaches the equilibrium, a. The values obtained for the high, low and overall (high and low) abundance areas were $\mathrm{b}=$ $0.0039 \pm 0.0042, b=0.0292 \pm 0.0045$ and $b=0.0099 \pm$ 0.0033 , respectively.

The goodness of fit of the Box-Lucas function alone does not conclusively demonstrate that the number of 
captures varies with the density of sticky traps showing a saturation trend to an equilibrium level. Nevertheless, the combined results for the low, high and overall abundance areas showing different and consistent equilibrium levels of $a$ and scales of $b$ for the Box-Lucas function, do suggest a saturation trend for the dependency of the number of Ae. aegypti females caught in sticky traps on the trap density.

In the low abundance areas, the effect of the density of sticky traps on the population size was more important, which caused the number of captures to reach values close to the Box-Lucas function equilibrium level more rapidly. For the high and overall abundance areas, the function fit did not reach values near an equilibrium level, which may be because the trap density was not sufficiently high to capture a relevant number of the existing Ae. aegypti females exposed to the set of sticky traps in the area. It is likely that both the high and overall abundance areas would show the same trend in reaching their respective equilibrium levels at sufficiently high trap densities. The values of $b$ corroborate this assumption, as $\mathrm{b}$ is higher for the low abundance areas. The $b$ parameter has units of area per sticky trap. It also provides an effective scale or range of the average depletion effect of the sticky traps on the mosquito population size per unit of area. Once b provides a scale, it also means that the interaction between the sticky traps and the population distributed in the low abundance area shows a long range compared to other cases. Upon considering the necessary corrections between the scales of surveillance times and gonotrophic cycles as well as between the scales of block dimensions and the dispersion of female mosquitoes, the values of a for the Box-Lucas function could be used to estimate the size of Ae. aegyp$t i$ female population for both low and high abundance areas. However, further studies should be conducted to confirm this assumption.

We found that the ability of the MosquiTRAPTM's to detect the presence of Ae. aegypti was dependent on the trap density per block. Installation of at least eight MosquiTRAPs ${ }^{\mathrm{TM}}$ per block resulted in over $90 \%$ positivity, at least under the conditions of this investigation. In contrast, at a density of one MosquiTRAP ${ }^{\mathrm{TM}}$ per block, which is considered a suitable density for $A e$. aegypti, positivity ranged between $31-50 \%$ for low- and high abundance areas, respectively. These results were similar to those reported by Fávaro et al. (2006), who verified that the sensitivity of MosquiTRAP ${ }^{\mathrm{TM}}$ (82.1\%) was similar to that of ovitraps (89.7\%) for detecting the presence of Ae. aegypti when using a density of four MosquiTRAPs $^{\text {TM }}$ (1 sticky trap at each end of the block) or one ovitrap per block, whereas the positivity of the sticky traps was lower than that of ovitraps when using the same density per block (1:1) (Gama et al. 2007, Honório et al. 2009a). However, MosquiTRAPs ${ }^{\mathrm{TM}}$ allow detection and monitoring of Ae. aegypti throughout the year and this method has been shown to be more sensitive than larval surveys (Gama et al. 2007). In addition, the entomological indices provided by collecting adult mosquitoes are considered to be strongly correlated with the risk of dengue transmission (Focks 2003, Fávaro et al. 2006, Eiras \& Resende 2009).
The effect of the MosquiTRAP ${ }^{\mathrm{TM}}$ density per house suggests that an increase from one to four MosquiTRAPS ${ }^{\mathrm{TM}}$ per house resulted in a higher capture rate for Ae. aegypti. This result corroborates those found by Craig et al. (2006), who evaluated the use of sticky traps in Cairns, Australia and found that the installation of up to eight traps per house (4 inside and 4 outside) resulted in a higher mean number of Ae. aegypti adult mosquitoes collected per house. Nevertheless, installing eight traps per house may lead to operational complications for vector control programs and homeowners may resist placing this number of traps in their dwellings. The fact that there was a smaller relative increase in capture rates when comparing densities of one and two sticky traps per block than between two-four traps suggests that the above effect of saturation to an equilibrium level for higher densities would also be observed at the house scale.

In the current study, in the high, low and overall abundance areas, a large variance was observed in the MFAI. A large variance would be expected if the sticky traps behaved, as we believe, showing a Poisson distribution-like pattern in the frequency of the number of captures. In fact, for a sufficiently large number of mosquitoes, considering captures as the desired event in a fixed time interval of observation, it can be expected that the frequency sticky trap of captures will exhibit a Poisson-like distribution characterised by a high frequency of zero captures and mean and variance values that are numerically similar. In contrast to the weekly monitoring programs occurring in Brazil, in the present study, many indices were averaged over a four-week time period. Therefore, we believe that a relevant portion of the variance obtained was due to the intrinsic variation of the Ae. aegypti population from the initial population size during the preliminary test to the longer period of four weeks used in the experiment.

When focusing on the variance in the MFAI across the various tested densities of MosquiTRAPs ${ }^{\mathrm{TM}}$, we observed a greater variance of the MFAI when lower trap densities (1-4 traps per block) were installed compared to high trap densities (8-64 traps per block). Nevertheless, the density of one trap per block was sufficient to produce MFAI values similar to those when one-64 MosquiTRAP per block were installed. Such a result is desirable, though it is not sufficient to establish that a density of one trap per block is a suitable indicator for monitoring Ae. aegypti populations.

Among the many desired attributes that an index should exhibit, the capability to distinguish between high and low-infestation areas is an important attribute. Based on the MFAI values obtained in the present investigation, we observed that for each trap density studied $(1,2,4,8,16,32$ and 64 traps/block) the variance and sensitivity of MosquiTRAP ${ }^{\mathrm{TM}}$ PMI affected the ability of the entomological index, the MFAI, to differentiate between high and low abundance areas of mosquitoes. A significant difference was observed between high and low abundance areas when these areas were compared regardless of the trap density. In contrast, at a density of 32 traps/block, the probability of separation was marginal and no significant difference was observed for densities ranging from one-16 traps/block. The sig- 
nificant difference observed between the high and low abundance areas when a density of 64 traps/block was used might be due to the impact of the trap density on the local mosquito population. A density of 64 traps/block may also have positively affected the separation of areas in the comparison test regardless of the trap density. The apparent inability of the MFAI to distinguish between high and low abundance areas might be due to the intrinsic variation in the Ae. aegypti population from the initial size during the preliminary analysis to the longer four-week period of our experiment, which produced different results compared to the MFAI calculated from the weekly monitoring programs currently in use. As several of the studies mentioned above have demonstrated the feasibility of adopting the MosquiTRAP ${ }^{\mathrm{TM}}$ and have shown its potential for monitoring Ae. aegypti females, further studies must be conducted to investigate the effects of the monitoring period against intrinsic changes in the Ae aegypti population size more precisely.

Using a set of concurrent characteristics is important to define which index and which trap density should be used to monitor sensitivity, variance, operational feasibility and particularly the purpose of setting the traps. The trap density should be defined based on program goals. For instance, if the objective is to detect the presence of Ae. aegypti in a particular block, our sensitivity results indicate that eight traps should be installed per block (1 trap/house) for a period of seven days. An investigation performed by Lourenço-de-Oliveira et al. (2008) detected a saturation trend after a period of seven days for ovitraps. However, in the present study, although there was a tendency to reach an equilibrium level when more than 16 traps per block were installed, a density of eight traps per block resulted in a sensitivity rate of approximately $90 \%$ (PMI), which may be used to accurately determine areas of high and low mosquito abundance. Despite the low sensitivity associated with a density of one MosquiTRAP ${ }^{\mathrm{TM}}$ per block, the use of this density for the continuous monitoring of Ae. aegypti in dengue control programs is acceptable, provided that the reference index for surveillance is the MFAI. Control program selection should take into account a sufficiently large set of contiguous and correlated blocks in clusters rather than a single block. Moreover, based on the variance tests, the MFAI values were similar for various trap densities per block, which has been demonstrated by other studies. The strategy of using one trap per block can substantially reduce the number of periodic visits by field workers, costs (for traps, lures and adhesive cards) and human resource requirements.

The simplest use of the PMI is to determine whether a vector is present or not, which is how it was used in the preliminary screening. The feasibility of employing the PMI for monitoring vector abundance is expected to be highly dependent on the applied monitoring frequency as well on the density of MosquiTRAPs ${ }^{\mathrm{TM}}$ used. When these parameters are set, this index would behave similarly to the MFAI, which would result in redundancy. Because the MFAHI behaved similarly for the total number of captures studied and the MFAHI corresponds only to the total number divided by the number of traps and by the number of houses over a given time, its definition is somewhat similar to that of the MFAI, which results in the MFAI being a sufficient index for use in monitoring programs. Therefore, we suggest applying MosquiTRAP ${ }^{\mathrm{TM}}$ inspection on a weekly basis for MFAI analysis at a density of one trap per block for continuous monitoring of Ae. aegypti in dengue control programs.

Once the number of MosquiTRAPs ${ }^{\mathrm{TM}}$ per block associated with the equilibrium level of Ae. aegypti females captured is better understood, higher densities of MosquiTRAPs $^{\mathrm{TM}}$ may be used for controlling the dengue vector as well for studying mosquito population sizes. A study combining analyses mosquito skipping oviposition behaviour and trap density is ongoing. Regis et al. (2008) used a modified ovitrap (3 paddles, adding Bacillus thuringiensis israelensis in the infusion of ovitrap) to control Ae. aegypti in the city of Recife (Brazil). The authors verified that using this modified ovitrap in the field for two months resulted in the collection of more than 7,000 eggs/ovitrap and they suggested that carrying out a massive egg collection combined with treatment of water containers with larvicides could be a promising strategy for vector control. Nevertheless, further studies should be performed to compare study sites with control areas where traps are absent as well as to carry out surveillance of adult mosquitoes to confirm the reduction of the Ae. aegypti population in a study area. Similar efforts have been applied in Australia (Ritchie et al. 2009) to suppress adult mosquito populations using a lethal ovitrap consisting of an oviposition trap containing stripe paper impregnated with insecticide. Accordingly, future studies using mass trapping need to be conducted with specific protocols to assess the effect of the number of MosquiTRAPs $^{\mathrm{TM}}$ on Ae. aegypti control. This technique has been used successfully for controlling insect pests in agriculture (Vilela \& Della Lúcia 2001, Choi et al. 2011), based on which adult insects might be effectively attracted and captured in large numbers using lures and traps. We have expectations that MosquiTRAP ${ }^{\mathrm{TM}}$ would be useful in designing a municipal dengue control program, with mass trapping activities being directed to areas with higher mosquito abundances.

\section{ACKNOWLEDGEMENTS}

To Dr Renato Assunção (ICEx-UFMG), Dr Neal Alexander (London School of Hygiene and Tropical Medicine), Dr Marcelo Burattini and Dr Eduardo Massad (USP), for their valuable suggestions, to Paola Eiras, for English review, to the reviewers, for their valuable contribution, to the health agents from the Regional Health West Secretariat of Belo Horizonte, trainees of the Laboratory of Chemical Ecology and Vectors of UFMG and Giovani Gonçalves Pontel, João Manuel Peixinho and Madson Neves (FUNASA-MG), for collaborating on the field work, and to Ecovec SA, for providing the sticky traps.

\section{REFERENCES}

Box GEP, Lucas HL 1959. Design of experiments for non-linear situations. Biometrika 46: 77-90.

Braga IA, Gomes AC, Nelson M, Mello RC, Bergamaschi DP, Souza JMP 2000. Comparação entre pesquisa larvária e armadilha de oviposição para detecção de Aedes aegypti. Rev Soc Bras Med Trop 33: 347-353. 
Braga IA, Valle D 2007. Aedes aegypti: histórico do controle no Brasil. Epidemiol Serv Saúde 16: 113-118.

Breteau H 1954. La fiève jaune en Afrique-Occidenatlle Française. Un aspect de la médecine préventive massive. Bull WHO 11: 453-481.

Choi HS, Kim GJ, Shin HJ 2011. Biocontrol of moth pests in apple Orchards: preliminary field study of application potential for mass trapping. Biotech Bioprocess Engineering 16: 153-157.

Connor ME, Monroe WM 1923. Stegomyia indices and their value in yellow fever control. Am J Trop Med Hyg 3: 9-19.

Craig RW, Sharron AL, Russell RC, Ritchie SA 2006. Optimizing ovitrap use for Aedes aegypti in Cairns, Queensland, Australia: effects of some abiotic factors on field efficacy. J Am Mosq Control Assoc 22: 635-640.

Eiras AE, Resende MC 2009. Preliminary evaluation of the "DengueMI" technology for Aedes aegypti monitoring and control. Cad Saude Publica 25 (Suppl.): S45-S58.

Facchinelli L, Valerio L, Pombi M, Reiter P, Costantini C, Della Torre A 2007. Development of a novel sticky trap for containerbreeding mosquitoes and evaluation of its sampling properties to monitor urban populations of Aedes albopictus. Med Vet Entomol 21: 183-195.

Faccioli G, Pasqualini E, Baronio P 1993. Optimal trap density in Cossus cossus (Lepidoptera: Cossidae) mass-trapping. J Econ Entomol 86: 850-853.

Fávaro EA, Dibo MR, Mondini A, Ferreira AC, Barbosa AAC, Eiras AE, Barata EAMF, Chiaravalloti-Neto F 2006. Physiological state of Aedes (Stegomyia) aegypti mosquitoes captured with MosquiTRAP $^{\mathrm{TM}}$ in Mirassol, São Paulo, Brazil. J Vector Ecol 31: 285-291.

Fávaro EA, Mondini A, Dibo MR, Barbosa AAC, Eiras AE, Chiaravalloti-Neto FC 2008. Assessment of entomological indicators of Aedes aegypti (L.) from adult and egg collections in São Paulo, Brasil. J Vector Ecol 33: 8-16.

Focks DA 2003. A review of entomological sampling methods and indicators for dengue vectors, World Health Organ, Gainesville, $40 \mathrm{pp}$.

Focks DA, Chadee DD 1997. Pupal survey: an epidemiologically significant surveillance method for Aedes aegypti; an example using data from Trinidad. Am J Trop Med Hyg 56: 159-167.

Gama RA, Silva EM, Silva IM, Resende MC, Eiras AE 2007. Evaluation of the sticky MosquiTRAP for detecting Aedes aegypti Linnaeus (Diptera: Culicidae) during the dry season in the district of Itapoã, Belo Horizonte, Minas Gerais, Brazil. Neotrop Entomol 36: 294-302.

Honório NA, Silva WC, Leite PJ, Gonçalves JM, Lounibos LP, Lourenço-de-Oliveira R 2003. Dispersal of Aedes aegypti and Aedes albopictus (Diptera: Culicidae) in an urban endemic dengue area in the state of Rio de Janeiro, Brazil. Mem Inst Oswaldo Cruz 98: 191-198.

Honório NA, Codeço CT, Alves FC, Magalhães MAFM, Lourençode-Oliveira R 2009a. Temporal distribution of Aedes aegypti in different districts of Rio de Janeiro, Brazil, measured by two types of traps. J Med Entomol 46: 1001-1014.

Honório NA, Nogueira RMR, Codeço CT, Carvalho MS, Cruz OG, Magalhães MAFM, Araújo JMG, Araújo ESM, Gomes MQ, Pinheiro LS, Pinel CS, Lourenço-de-Oliveira R 2009b. Spatial evaluation and modeling of dengue seroprevalence and vector density in Rio de Janeiro, Brazil. PLoS Negl Trop Dis 3: e545.

IBGE - Instituto Brasileiro de Geografia e Estatística 2000. [homepage on the Internet]. Censo demográfico 2000. [updated 2000; cited 2009 Aug 18]. Available from: ibge.gov.br/home/estatistica/ populacao/censo2000/default.shtm.

Iga M 1982. Density of methyl eugenol traps for efficient trapping of adult males of oriental fruit fly Dacus dorsalis (Diptera: Te- phritidae) on Ogasawara Island Japan. Jpn J Appl Entomol Zool 26: 172-176.

Lagrotta MTF, Silva WC, Santos RS 2008. Identification of key areas for Aedes aegypti through geoprocessing in Nova Iguaçu, Rio de Janeiro state, Brazil. Cad Saude Publica 24: 70-80.

Lourenço-de-Oliveira R, Lima JBP, Peres R, Alves FC, Eiras AE, Codeço CT 2008. Comparison of different uses of adult traps and ovitraps for assessing dengue vector infestation in endemic areas. J Am Mosq Control Assoc 24: 387-392.

Maciel-de-Freitas R, Eiras AE, Lourenço-de-Oliveira R 2006. Field evaluation of effectiveness of the BG-Sentinel, a new trap for capturing adult Aedes aegypti (Diptera: Culicidae). Mem Inst Oswaldo Cruz 101: 321-325.

Maciel-de-Freitas R, Peres RC, Alves F, Brandolini MB 2008. Mosquito traps designed to capture Aedes aegypti (Diptera: Culicidae) females: preliminary comparison of Adultrap, MosquiTRAP and backpack aspirator efficiency in a dengue-endemic area of Brazil. Mem Inst Oswaldo Cruz 103: 602-605.

MS - Ministério da Saúde 2002. Fundação Nacional de Saúde. Ministério da Saúde. Programa Nacional de Controle da Dengue - PNCD, FUNASA, Brasília, 32 pp.

MS - Ministério da Saúde 2009. Diretrizes nacionais para prevenção e controle de epidemias de dengue, Secretaria de Vigilância em Saúde/Departamento de Vigilância Epidemiológica, Brasília, 160 pp.

Ordónez-Gonzalez JG, Mercado-Hernandez R, Florez-Suarez AE, Fernandez-Salas I 2001. The use of sticky ovitraps to estimate dispersal of Aedes aegypti in Northeastern México. J Am Mosq Control Assoc 17: 93-97.

PBH - Prefeitura de Belo Horizonte 2009. [homepage on the Internet] Distrito Sanitário Oeste. [updated 2009; cited 2009 Aug 18]. Available from: portalpbh.pbh.gov.br/pbh/ecp/comunidade. do?app=regionaloeste.

Regis L, Monteiro AM, Melo-Santos MAV, Silveira Jr JCS, Furtado AF, Acioli RV, Santos GM, Nakazawa M, Carvalho MS, Ribeiro Jr PJ, Souza WV 2008. Developing new approaches for detecting and preventing Aedes aegypti population outbreaks: basis for surveillance, alert and control system. Mem Inst Oswaldo Cruz 103: 50-59.

Reiter P, Amador MA, Anderson RA, Clark GG 1995. Short report: dispersal of Aedes aegypti in an urban area after blood feeding as demonstrated by rubidium-marked eggs. Am J Trop Med Hyg 52: $177-179$.

Ritchie SA, Rapley LP, Williams CW, Johnson PH, Larkman M, Silcock RM, Long SA, Russell RC 2009. A lethal ovitrap-based mass trapping scheme for dengue control in Australia. I. Public acceptability and performance of lethal ovitraps. Med Vet Entomol 23: 295-302.

Russell RC, Ritchie SA 2004. Surveillance and behavioural investigations of Aedes aegypti and Aedes polynesiensis in Moorea, French Polynesia, using a sticky ovitrap. J Am Mosq Control Assoc 20: $370-375$.

Sokal RR, Rolf FJ 1995. Biometry: the principles and practice of statistics in biological research, 3rd. ed., WH Freeman, New York, 887 pp.

Teixeira MG, Costa MCN, Barreto ML, Mota E 2005. Dengue and dengue hemorrhagic fever epidemic in Brazil: what research is needed based on trends, surveillance and control experiences? Cad Saude Publica 21: 1307-1315.

Vilela EF, Della Lúcia TMC 2001. Introdução aos semioquímicos e terminologia. In EF Vilela, TMC Della Lucia, Feromônios de insetos - Biologia, química e emprego no manejo de pragas, 2nd ed., Holos, Ribeirão Preto, p. 9-206.

WHO - World Health Organization 2006. Scientific working group. Report on dengue, TDR/SWG/08, WHO, Geneva, 168 pp. 